\title{
In vivo evaluation of the anti-infection potential of gentamicin-loaded nanotubes on titania implants
}

\author{
This article was published in the following Dove Press journal: \\ International Journal of Nanomedicine \\ 19 May 2016 \\ Number of times this article has been viewed
}

\author{
Ying Yang' \\ Hai-yong Ao' \\ Sheng-bing Yang' \\ Yu-gang Wang' \\ Wen-tao Lin $^{2}$ \\ Zhi-feng Yu' \\ Ting-ting Tang' \\ 'Shanghai Key Laboratory of \\ Orthopedic Implants, Department \\ of Orthopedic Surgery, Shanghai \\ Ninth People's Hospital, Shanghai \\ Jiao Tong University, School of \\ Medicine, Shanghai, ${ }^{2}$ Department \\ of Orthopedics, Fuzhou Second \\ Hospital, Affiliated Hospital of Xiamen \\ University, Fuzhou, People's Republic \\ of China
}

Correspondence: Ting-ting Tang Shanghai Key Laboratory of Orthopedic Implants, Department of Orthopedic Surgery, Shanghai Ninth People's Hospital, Shanghai Jiao Tong University, School of Medicine, 639 Zhizaoju Road, Shanghai 2000II, People's Republic of China

Tel +862 2I 2327 II 33

$\mathrm{Fax}+862163137020$

Email ttt@sjtu.edu.cn

\begin{abstract}
Titanium-based implants have been widely used in orthopedic surgery; however, failures still occur. Our in vitro study has demonstrated that gentamicin-loaded, $80 \mathrm{~nm}$ diameter nanotubes possessed both antibacterial and osteogenic activities. Thus, the aim of this study was to further investigate the in vivo anti-infection effect of the titanium implants with gentamicin-loaded nanotubes. Thirty-six male Sprague Dawley rats were used to establish an implant-associated infection model. A volume of $50 \mu \mathrm{L}$ Staphylococcus aureus suspension $\left(1 \times 10^{5} \mathrm{CFU} / \mathrm{mL}\right)$ was injected into the medullary cavity of the left femur, and then the titanium rods without modification ( $\mathrm{Ti}$ ), titanium nanotubes without drug loading (NT), and gentamicinloaded titanium nanotubes (NT-G) were inserted with phosphate-buffered saline-inoculated Ti rods as a blank control. X-ray images were obtained 1 day, 21 days, and 42 days after surgery; micro-computed tomography, microbiological, and histopathological analyses were used to evaluate the infections at the time of sacrifice. Radiographic signs of bone infection, including osteolysis, periosteal reaction, osteosclerosis, and damaged articular surfaces, were demonstrated in the infected Ti group and were slightly alleviated in the NT group but not observed in the NT-G group. Meanwhile, the radiographic and gross bone pathological scores of the NT-G group were significantly lower than those of the infected Ti group $(P<0.01)$. Explant cultures revealed significantly less bacterial growth in the NT-G group than in the Ti and NT groups $(P<0.01)$, and the NT group showed decreased live bacterial growth compared with the Ti group $(P<0.01)$. Confocal laser scanning microscopy, scanning electron microscopy, and histopathological observations further confirmed decreased bacterial burden in the NT-G group compared with the Ti and NT groups. We concluded that the NT-G coatings can significantly prevent the development of implant-associated infections in a rat model; therefore, they may provide an effective drug-loading strategy to combat implant-associated infections in clinic.

Keywords: titanium nanotubes, gentamicin, implant-associated infection, animal model
\end{abstract}

\section{Introduction}

Currently, implant-associated infection is one of the critical causes of implant failures in orthopedic surgery. ${ }^{1-3}$ Titanium and titanium alloys are the most widely used implant materials and have good biocompatibility and excellent mechanical properties. ${ }^{4,5}$ However, these implant surfaces also favor bacterial adhesion, colonization, and biofilm formation, and there is a competition of initial adhesion to the implant surface between bacteria and osteoprogenitors. ${ }^{6,7}$ One study of 46,113 intramedullary nail operations demonstrated that the overall infection rates of humerus, femur, and tibia fractures were $1.0 \%, 0.8 \%$, and $1.5 \%$, respectively. ${ }^{8}$ Therefore, good antibacterial properties and osteoblast activity are the key points involved in implant material manufacturing.

The systemic administration of antibiotics and the removal of intramedullary nails are two conventional approaches applied in the treatment of implant-associated infection, 
which can compromise patient compliance and lead to severe complexities, such as systemic toxicity, osteomyelitis, septic arthritis, and bone ununion. ${ }^{9,10}$ Moreover, the infection rate reaches up to $33 \%$ in spite of the use of external fixation. ${ }^{11}$ Since the initial report on the fabrication of titania nanotube (TNT) arrays generated over a Ti surface by a simple and adjustable electrochemical anodization process in $1999,{ }^{12}$ they have been extensively explored as an innovative nanoengineering technology to mitigate the side effects of systemic drug administration. ${ }^{9,13,14}$ Meanwhile, TNT arrays have been utilized to form new drug-eluting implants based on localized drug delivery due to their high loading capability, tunable drug-releasing performance, and excellent biocompatibility. ${ }^{15}$ It has been widely shown that antibiotic-loaded TNTs are able to reduce bacterial adhesion, while retaining or improving normal osteoblast adhesion and differentiation. ${ }^{13,16-19}$ Various metal cations that possess antibacterial properties, such as Ag, $\mathrm{Zn}$, and $\mathrm{Cu}$, have been introduced into TNT arrays to enhance the antibacterial activity of TNT-based Ti implants. ${ }^{20-24} \mathrm{Nev}-$ ertheless, their potential toxicity and antibacterial durability have yet to be improved and investigated.

The success of osteoblasts versus bacteria in the implant surface adhesion competition has been shown to be significant for the deposition of bone matrix onto the biomaterials. ${ }^{25}$ Thus, the inhibition of initial bacterial adhesion and colonization is of importance to implant osseointegration. The nano-featured surface topographies of the TNT-based implants have been widely investigated as a potential approach for selectively enhancing desirable osteoblast functions while simultaneously inhibiting bacterial adhesion. Some reports, including our previous in vitro study, have demonstrated that $80 \mathrm{~nm}$-diameter TNTs produced the best antibacterial activity of all the surface treatment parameters tested. ${ }^{16,26,27}$ Another in vivo study showed that $70 \mathrm{~nm}$-diameter TNTs significantly improved the bone-implant contact and osseointegration-related gene expression levels when compared with the other tested titanium implants. ${ }^{28}$

Gentamicin, which is an aminoglycoside antibiotic, has been widely used in the treatment of implant-associated infections. Gentamicin-loaded nanotubes and polymer coatings have been shown in various studies to effectively inhibit bacterial adhesion while retaining good osteoinductivity. ${ }^{13,29}$ Meanwhile, our in vitro research revealed that gentamicinloaded nanotubes (NT-G) and nanotubes with no drug loading (NT), especially those with diameters of $80 \mathrm{~nm}$, significantly improved the antimicrobial effects and osteogenic activity of the implant surface. ${ }^{16,26}$ However, there have also been several reports indicating that the nanotubular surfaces increased bacterial adhesion compared with conventional and nanorough surfaces due to the presence of fluorine and amorphous crystallinity. ${ }^{30,31}$ Therefore, it is of great importance to evaluate the in vivo anti-infection effect and reliability of the drug-loaded nanotube coating. In addition, implant-related infection model in rats, which could be used to simulate the tibia or femur implant-related intramedullary infection in clinics, have been widely used to verify the treatment effect of implant with antibacterial properties in vivo. ${ }^{29,32}$ This study was designed to further investigate the effects of gentamicin-loaded nanotubes on the surface of titanium implants on experimental infection in a rat femur implant-related infection model using a standard Staphylococcus aureus (ATCC 25923) strain inoculation.

\section{Materials and methods Preparation of gentamicin-loaded, nanotube-coated titania rods}

The gentamicin-loaded, nanotube-coated titania rods were fabricated as described previously. ${ }^{9,16,33}$ In brief, the titania rods (20 $\mathrm{mm}$ in length and $1.5 \mathrm{~mm}$ in diameter) were chemically polished in a mixed solution including $\mathrm{HF}$ and $\mathrm{HNO}_{3}$ $\left(\mathrm{V}\left[\mathrm{HNO}_{3}\right]: \mathrm{V}[\mathrm{HF}]=1: 1\right.$ ) for 60 seconds, and then TNTs ( $80 \mathrm{~nm}$ in diameter and $2 \mu \mathrm{m}$ in length) were prepared over the surface of the rods after the anodization in the electrolyte (0.5 wt \% $\mathrm{NH}_{4} \mathrm{~F}$ and $10 \mathrm{vol} \%$ distilled water in ethylene glycol) for 1 hour at a constant voltage of $25 \mathrm{~V}$. Then, the rods were cleaned with deionized water and dried at room temperature for subsequent drug loading. A gentamicin (Sigma-Aldrich Co., St Louis, MO, USA) phosphate-buffered saline (PBS) solution of $100 \mathrm{mg} / \mathrm{mL}$ was prepared and loaded into the TNTs by a lyophilization and vacuum-drying method. ${ }^{13}$ The rods were rotated after each step to ensure even drug loading. Then, after the completion of the drug-loading procedure, the surfaces of the rods were gently rinsed with PBS to remove excess drug from the surface. Titania rods without modification (Ti) and nanotube-coated titania rods (NT) with uniform size were used as controls; the morphology of the nanotubes with or without drug-loading was shown in our previous report. ${ }^{16}$ All prepared rods were sterilized with $25 \mathrm{kGy}$ of ${ }^{60} \mathrm{Co}$ irradiation before in vivo implantation.

\section{Preparation and characterization of bacteria} S. aureus (ATCC 25923) was purchased in freeze-dried form from the American Type Culture Collection (Manassas, VA, USA). S. aureus (ATCC 25923) has been proved to be able to form biofilm. ${ }^{34}$ Meanwhile, this strain exhibits significant susceptibility to gentamicin (minimum inhibitory concentration, $1 \mu \mathrm{g} / \mathrm{mL}$ ), as determined by a microtiter broth dilution method 
as previously reported. ${ }^{35}$ Then, the bacteria were suspended in tryptone soy broth (TSB) solution at a concentration of $5 \times 10^{8} \mathrm{CFU} / \mathrm{mL}$ after overnight culture according to a McFarland standard protocol (Beijing Zhecheng Science and Technology Co., Ltd, Beijing, People's Republic of China). For the in vivo study, the concentration of the bacteria suspension was diluted to $1 \times 10^{5} \mathrm{CFU} / \mathrm{mL}$ in TSB solution.

\section{Surgical procedures}

All experimental procedures (including surgery and drug administration) were approved and performed in accordance with the guidelines of the Animal Ethics Committee of Shanghai Ninth People's Hospital. Thirty-six specified pathogen free (SPF) grade 8-week-old male Sprague Dawley rats (weighing 421.77 $\pm 62.72 \mathrm{~g}$ ) were used and randomly assigned to four independent groups. The surgical procedures were similar to the previously reported femur implant-related infection model.$^{32}$ In brief, the rats were initially anesthetized using an intraperitoneal injection of $1 \%$ pentobarbital sodium (100 mg/kg body weight). The left knee of each animal was shaved and cleaned with $70 \%$ ethanol prior to the procedure, and then the left knee was opened via a middle parapatellar incision. The femoral medullary cavity was opened at the middle of the femoral trochlear after the dislocation of the patella and then was gradually reamed with Kirschner wires up to a diameter of $1.5 \mathrm{~mm}$. Subsequently, $50 \mu \mathrm{L}$ of either PBS or TSB containing ATCC 25923 bacteria at a concentration of $1 \times 10^{5} \mathrm{CFU} / \mathrm{mL}$ was injected into the medullary cavity using a micropipette. Then, a prepared Ti, NT, or NT-G rod was inserted (Table 1). The surgical site closure was performed layer by layer after the opening was sealed with bone wax. The rats were kept in separated cages and allowed to eat and drink ad libitum. The animals were sacrificed after 6 weeks. No antibiotic was administered postsurgery.

\section{Clinical assessment}

All animals in those four groups were monitored on the day of surgery and 3 days, 7 days, 14 days, 21 days, 28 days, 35 days, and 42 days after surgery. Body temperature and

Table I Animal experimental groups

\begin{tabular}{llll}
\hline Group & Number $(\mathbf{n})$ & Implant & Inoculation \\
\hline I & 9 & Ti rod & S. aureus $10^{5} \mathrm{CFU} / 50 \mu \mathrm{L}$ \\
II & 9 & NT rod & S. aureus $10^{5} \mathrm{CFU} / 50 \mu \mathrm{L}$ \\
III & 9 & NT-G rod & S. aureus $10^{5} \mathrm{CFU} / 50 \mu \mathrm{L}$ \\
IV & 9 & Ti rod & PBS $/ 50 \mu \mathrm{L}$ \\
\hline
\end{tabular}

Note: $\mathrm{Ti}$, titanium without modification.

Abbreviations: NT, nanotubes; NT-G, gentamicin-loaded nanotubes; PBS, phosphatebuffered saline; S. aureus, Staphylococcus aureus. weight were selected as general index observations and evaluated from all the nine animals in each group. ${ }^{36}$ Weight was measured on a precision scale (TCS, Shanghai, People's Republic of China), and body temperature was determined by a veterinary digital infrared thermometer (HRQ-S60; Zhengzhou, People's Republic of China). Local clinical signs of infection included knee joint swelling, skin exudation, and other inflammatory signs.

\section{Radiographic evaluation}

Femur and knee joint lateral radiographs were obtained at 1 day, 21 days, and 42 days postsurgery. Radiographic manifestations were assessed on the basis of a modified scoring system, ${ }^{36,37}$ as follows: 1, periosteal reaction; 2, osteolysis; 3, soft tissue swelling; 4, deformity; 5 , general impression; 6 , spontaneous fracture; and 7, sequestrum formation. Parameters 1-5 were scored as follows: 0, absent; 1, mild; 2, moderate; and 3, severe. Parameters 6 and 7 were recorded as 0 (absent) or 1 (present). $\mathrm{X}$-rays of the femurs and knee joints in all the groups were read and interpreted in a blind manner by a radiologist unaware of the grouping characteristics and inocula. The modified femurs were harvested and evaluated using a high-resolution micro-CT (Scanco Medical, $\mu$ CT 80, Brüttisellen, Switzerland) at an isometric resolution of $20 \mu \mathrm{m}(70 \mathrm{kV}$ and $130 \mu \mathrm{A}$ radiation source with $0.5 \mathrm{~mm}$ aluminum filter). Three-dimensional, high-resolution images obtained from overall, longitudinal and transverse sections, bone volume/total volume, and the cortical bone mineral density of the rat femurs were reconstructed and analyzed using the software provided by the manufacturer (Image Processing Language, v4.29d; Scanco Medical AG, Brüttisellen, Switzerland). The evaluation of the X-ray and micro computed tomography (CT) manifestations was obtained from five animals randomly selected in each group.

\section{Evaluation of gross bone pathology}

Three rats in each group were randomly sacrificed at 42 days postsurgery for evaluating gross bone pathology; their femurs were aseptically harvested, and the intramedullary rods were removed. The explanted rods were fixed in $4 \%$ buffered formaldehyde for 4 hours at a temperature of $4^{\circ} \mathrm{C}$ for subsequent confocal laser scanning microscopy (CLSM) and scanning electron microscopy (SEM). Femurs from each group were then cut sagittally for gross pathological observation, and those samples were subsequently used for histopathological evaluation. The gross bone pathology scores were assessed using a grading system: ${ }^{36,38} 0$, absence of abscess, sequestrum, active bone formation, and erythema; 1, minimal erythema without abscess or evidence of new bone formation; 
2 , erythema accompanied by widening of the shaft and new bone formation in the bone shaft; 3 , abscess with new bone formation, sinus tract drainage, periosteal reaction, or grossly purulent exudate; and 4, severe bone resorption, abscess, and diaphyseal or total tibial involvement. The scores of the gross bone pathology were evaluated and obtained by a pathologist blinded to the samples' grouping characteristics.

\section{Microbiological evaluation}

On the day of sacrifice, the remaining three rods were aseptically explanted and rolled over tryptone soy agar (TSA) plates to semi-quantify bacteria implant adhesion. The rods were placed in $5 \mathrm{~mL}$ PBS and then sonicated in an ultrasonic bath at 150 W (B3500S-MT, Shanghai, People's Republic of China) operating at a frequency of $50 \mathrm{~Hz}$ for 5 minutes, followed by rapid vortex mixing (Vortex-Genie 2; Scientific Industries, New York, NY, USA) at maximum power for 1 minute to dislodge the adhered bacteria thoroughly. ${ }^{29,32,39}$ The solutions collected after ultrasonication were then serially diluted tenfold and were plated in triplicate onto TSA plates; the plates were then incubated at a temperature of $37^{\circ} \mathrm{C}$ for 24 hours. The number of colonies on the TSA plates was counted, and the final number of CFUs on each rod was equal to the number of colonies multiplied by the dilution ratio. Following implant removal, the three femurs of each group were weighed, chilled with liquid nitrogen, crushed into fragments, and pulverized in a sterile bone mill. ${ }^{36}$ The bone powder was homogeneously vortexed in $5 \mathrm{~mL}$ PBS for 2 minutes. After centrifuging at 10,000 rpm for 20 seconds, $50 \mu \mathrm{L}$ of the supernatant was drawn and serially diluted tenfold. The bacteria quantity in each bone powder was analyzed and expressed relative to the femur weight (CFU/g femur) using the spread plate method mentioned earlier.

CLSM and SEM were used for direct observation of biofilm formation on the different rods of the four groups. After the 4\% buffered formaldehyde fixation, the rods were stained in a fresh six-well plate (CoStar; Corning Incorporated, New York, NY, USA) with $500 \mu \mathrm{L}$ of combination dye (LIVE/DEAD BacLight Viability Kits, L7012; Thermo Fisher Scientific, Waltham, MA, USA) and visualized by CLSM (Leica TCS SP8; Leica Microsystems, Wetzlar, Germany). The viable bacteria with intact cell membranes appeared fluorescent green, whereas nonviable bacteria with damaged cell membranes appeared fluorescent red. The three-dimensional images were acquired from random rod positions. After the CLSM observations, the rods were then dehydrated by a series of graded ethanol solutions $(30 \%, 50 \%, 70 \%, 80 \%, 90 \%$, and $100 \%)$ for 10 minutes each. Subsequently, the rods were examined using a scanning electron microscope (SEM; JEOL JSM-6700F, Tokyo, Japan) after critical-point drying and gold sputter-coating.

\section{Bone histopathology}

For histopathological evaluation, six medial femur halves after the gross observation and three intact femurs without rods after the micro-CT detection from each group were fixed in $4 \%$ buffered formaldehyde for 48 hours and decalcified for 7-10 days using a Rapid Decalcifier (DeCa DX-1000; ProCure Medical Technology Co. Ltd, Kwun Tong, Hong Kong). The samples were then embedded in paraffin and cut using a microtome (CUT 6062; SLEE Medical, Mainz, Germany) to obtain $5 \mu \mathrm{m}$ longitudinal and transverse sections. Hematoxylin and eosin and Masson's trichrome staining were used to assess morphology, and Giemsa staining was used to assess bacterial contamination. Meanwhile, after fixation and graded dehydration, three undecalcified femurs containing implants were embedded in methyl methacrylate (MMA) for infiltration and polymerization. The embedded specimens were transversely cut at the middle of the left femur (Leica SP1600 cutting equipment), the cut surface was glued to a slide, and the sections were ground to a thickness of $\sim 50 \mu \mathrm{m}$. The undecalcified sections containing implants were stained with van Gieson to observe the morphology of cortical bone around the implants.

\section{Statistical analysis}

All data are expressed as the mean $\pm \mathrm{SD}$. A one-way analysis of variance and least significant difference tests were utilized to assess differences in gross bone pathology scores and body weights and temperatures between groups. Nonparametric tests for independent samples (the Mann-Whitney $U$ test) were performed for comparison of the radiographic scores and CFUs from microbiological evaluations between groups. $P<0.05$ was defined as statistically significant. All statistical analyses were performed using SPSS software (v19.0, IBM Corporation, Armonk, NY, USA).

\section{Results}

\section{Clinical assessment}

No deaths or severe systemic complications occurred during the 6-week postsurgery follow-up period. Four rats in group I and three rats in group II showed slight swelling around the surgical sites on days 2-5 after surgery; however, the remaining animals in groups I and II and all the animals in group III exhibited no evident exudation or suppuration.

The body weights of all animals increased gradually from day 1 to day 42, and there were no significant differences in the weight changes among the four groups $(P>0.05)$. 
Table 2 Mean body weight and temperature values of the different groups

\begin{tabular}{|c|c|c|c|c|c|c|c|c|}
\hline Groups & Day 0 & Day 3 & Day 7 & Day I4 & Day 21 & Day 28 & Day 35 & Day 42 \\
\hline \multicolumn{9}{|l|}{ Temperature $\left({ }^{\circ} \mathrm{C}\right)$} \\
\hline $\mathrm{Ti}+\mathrm{S}$. aureus & $37.3 \pm 0.3$ & $37.0 \pm 0.4$ & $37.3 \pm 0.4$ & $37.3 \pm 0.3$ & $37.2 \pm 0.3$ & $37.1 \pm 0.4$ & $37.2 \pm 0.3$ & $37.1 \pm 0.5$ \\
\hline NT + S. aureus & $37.0 \pm 0.3$ & $37.2 \pm 0.5$ & $37.0 \pm 0.7$ & $37.0 \pm 0.4$ & $37.2 \pm 0.3$ & $36.9 \pm 0.7$ & $37.0 \pm 0.4$ & $37.1 \pm 0.4$ \\
\hline NT-G + S. aureus & $37.3 \pm 0.3$ & $37.1 \pm 0.4$ & $37.3 \pm 0.3$ & $37.2 \pm 0.4$ & $37.0 \pm 0.5$ & $37.2 \pm 0.4$ & $37.0 \pm 0.5$ & $37.2 \pm 0.3$ \\
\hline $\mathrm{Ti}+\mathrm{PBS}$ & $37.2 \pm 0.3$ & $37.3 \pm 0.5$ & $36.9 \pm 0.4$ & $37.3 \pm 0.3$ & $37.0 \pm 0.4$ & $37.1 \pm 0.5$ & $37.3 \pm 0.2$ & $37.2 \pm 0.3$ \\
\hline \multicolumn{9}{|l|}{ Body weight (g) } \\
\hline $\mathrm{Ti}+\mathrm{S}$. aureus & $436.1 \pm 51.8$ & $439.8 \pm 52.1$ & $445.2 \pm 53.0$ & $451.9 \pm 52.7$ & $461.5 \pm 55.8$ & $473.2 \pm 56.9$ & $483.6 \pm 51.9$ & $496.7 \pm 51.0$ \\
\hline $\mathrm{NT}+\mathrm{S}$. aureus & $465.4 \pm 56.9$ & $467.8 \pm 55.6$ & $471.5 \pm 52.4$ & $476.3 \pm 57.3$ & $482.3 \pm 52.1$ & $488.0 \pm 50.0$ & $496.4 \pm 53.1$ & $506.9 \pm 56.3$ \\
\hline NT-G + S. aureus & $383.1 \pm 49.0$ & $384.3 \pm 47.0$ & $386.7 \pm 47.3$ & $396.3 \pm 47.7$ & $4 \mid 3.1 \pm 52.0$ & $423.1 \pm 50.2$ & $430.3 \pm 51.0$ & $443.5 \pm 52.3$ \\
\hline $\mathrm{Ti}+\mathrm{PBS}$ & $402.5 \pm 80.0$ & $402.6 \pm 77.7$ & $405.2 \pm 77.9$ & $416.2 \pm 79.2$ & $425.9 \pm 81.8$ & $433.9 \pm 81.3$ & $442.4 \pm 77.9$ & $452.0 \pm 74.6$ \\
\hline
\end{tabular}

Note: Ti, titanium without modification.

Abbreviations: NT, nanotubes; NT-G, gentamicin-loaded nanotubes; PBS, phosphate-buffered saline; S. aureus, Staphylococcus aureus.

The body temperatures of all animals remained consistent and normal, with no significant differences between the groups $(P>0.05)$ (Table 2). The gradually increased body weights and relatively stable temperatures demonstrated in all the groups indicated that the bone infections were localized intramedullary or around the femurs, which may be the primary cause of bone-related infection diagnosis difficulty.

\section{Radiographic evaluation}

Radiographic signs of obvious osteolysis and slight periosteal reactions around the distal femurs in all the animals of groups I and II were observed by X-ray after 3 weeks postsurgery; increases in destruction of articular surfaces, osteosclerosis, and deformities were also observed. None of the X-ray images obtained from groups III and IV exhibited obvious signs of bone infection, including osteolysis and periosteal reactions (Figure 1A). Furthermore, the micro-CT analyses were used to corroborate the radiographic observations mentioned earlier on the day of sacrifice (Figure 1B). Obvious implant loosening and porous changes in the femoral cortical bones of groups I and II were observed, whereas good implant osseointegration and cortical integrity were observed in groups III and IV.

The quantitative analysis of the X-ray images in Figure $1 \mathrm{C}$ showed that the radiographic scores increased gradually after surgery for groups I and II $(P<0.01)$, whereas the scores slightly increased at 3 weeks and then gradually decreased at 6 weeks in group III $(P>0.05)$. Meanwhile, the scores remained steady in group IV $(P>0.05)$ over the course of the follow-up period. Groups I and II had significantly higher mean scores than the other two groups $(P<0.01)$ at 3 weeks and 6 weeks postsurgery, and group II exhibited lower mean scores than group I at the time of sacrifice $(P<0.05)$. Meanwhile, there were no significant differences between groups III and IV $(P>0.05)$. Moreover, the bone volume/total volume (Figure 1D) and the mean cortical bone mineral density (Figure 1E) of groups III and IV were significantly higher than those of the other two groups $(P<0.01)$, which were analyzed by the provided software after micro-CT reconstruction.

\section{Gross bone pathology}

As demonstrated in Figure 2A, animals in groups I and II showed obvious clinical signs of pyogenic infections, whereas animals in groups III and IV appeared to be free of infection, except for slight local fibroses in the medullary spaces. Evident intramedullary pus formation, periosteal reactions, osteolytic lesions, and bone deformities were the main clinical symptoms observed in group I; there was also local pus formation in group II. The mean gross bone pathological scores of groups I-IV were $3.33 \pm 1.16,2.67 \pm 0.58$, $1.00 \pm 0.00$, and $0.67 \pm 0.58$, respectively (Figure $2 \mathrm{~B}$ ). The mean score of group I was significantly higher than those of groups III and IV $(P<0.01)$. The mean score of group II was also significantly higher than those of group III $(P<0.05)$ and group IV $(P<0.01)$, respectively. Meanwhile, there were no significant differences between groups I and II or between groups III and IV $(P>0.05)$.

\section{Microbiological evaluation}

All cultures from group IV remained absolutely sterile after 24 hours of incubation. The quantity of bacteria colonies detached from the rods showed the following trend: group I $>$ group II $>$ group III (Figure 3A). Meanwhile, CLSM observation was utilized to determine the various bacterial loads among the four groups. In accordance with the results presented earlier, the implants in group IV showed no bacterial growth. As demonstrated in Figure 3B, live bacteria were indicated by green fluorescence and dead bacteria were indicated by red fluorescence. There were clear differences 
A

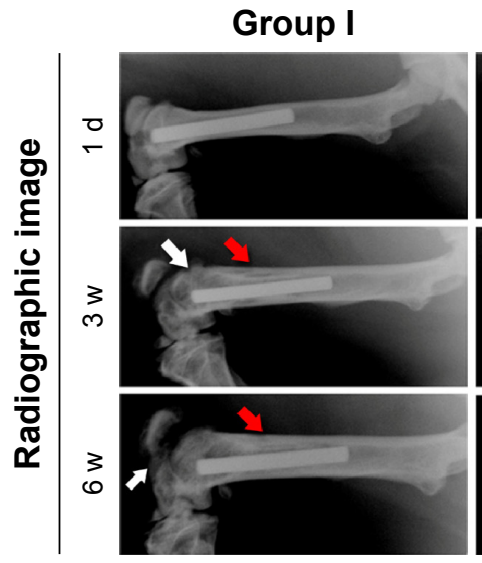

B

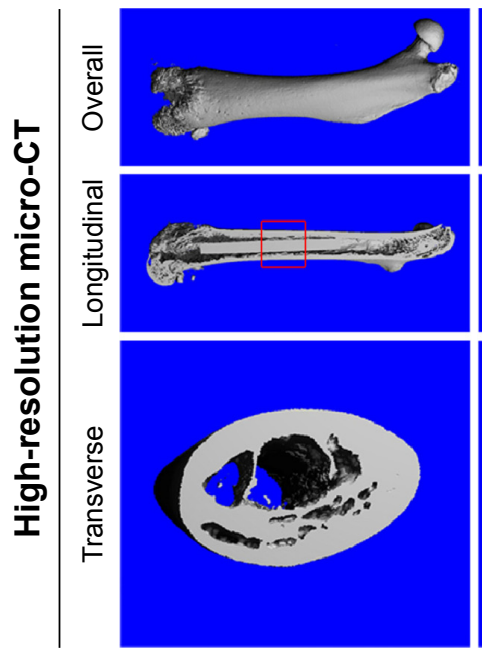

C

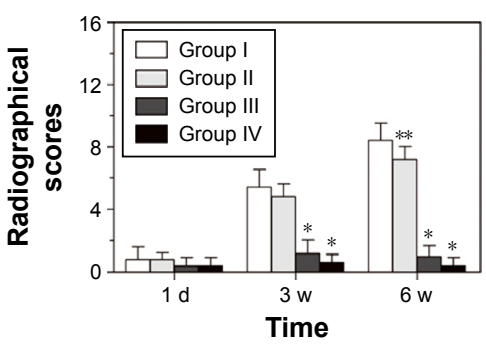

Group II
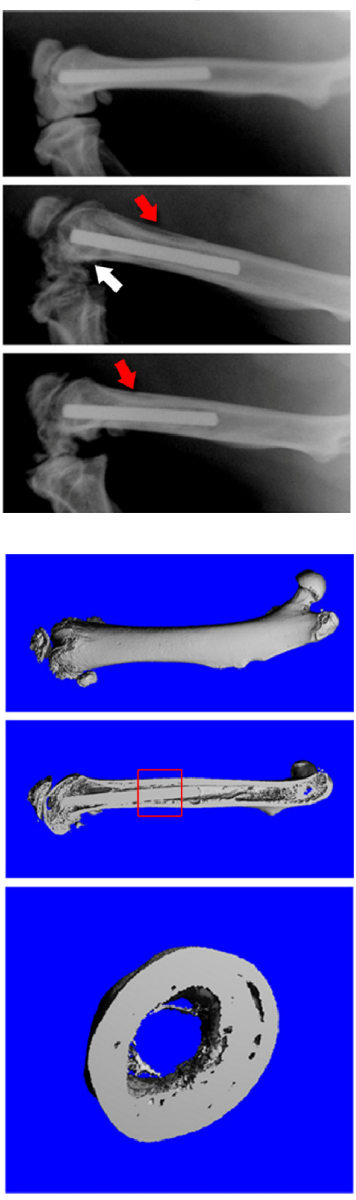

D

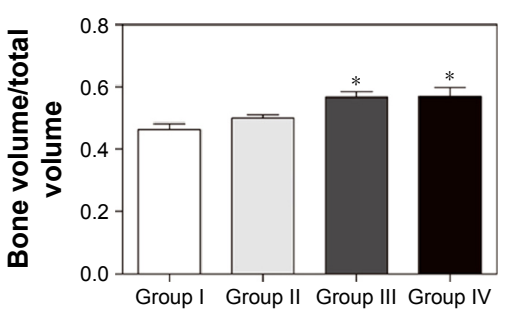

Group III
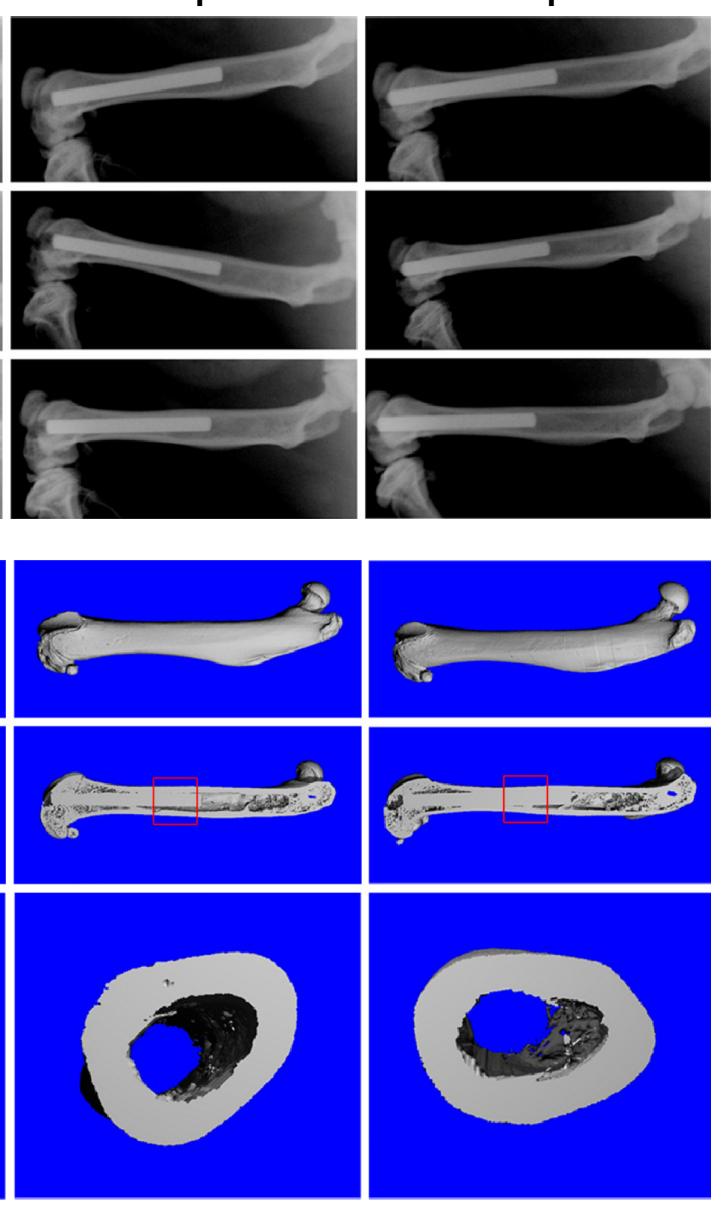

E

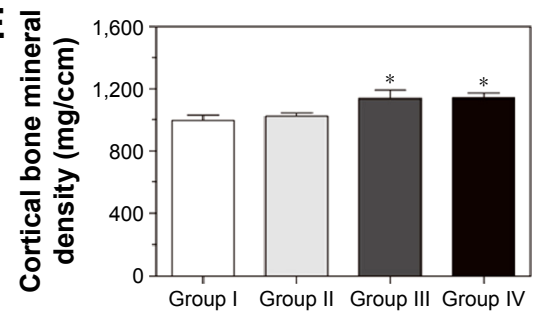

Figure I Radiographic images and analysis at I day, 3 weeks, and 6 weeks.

Notes: (A) Lateral X-rays of the left femur. The red arrows indicate osteolysis and slight periosteal reactions around the distal femurs, while the white arrows indicate articular surface damage, osteosclerosis, and deformities. (B) Three-dimensional micro-CT images of the left femur at the time of sacrifice. The micro-CT evaluations of the middle femurs were confined to the red square region. (C) Radiographic scores of the X-ray images. *Denotes a significant difference compared with groups I and II $(P<0.0 \mathrm{I}, \mathrm{n}=5)$. **Denotes a significant difference compared with group I $(P<0.05, \mathrm{n}=5)$. (D) Bone volume/total volume and $(\mathrm{E})$ cortical bone mineral density of the selected regions of the left femurs evaluated by micro-CT. *Denotes a significant difference compared with groups I and II $(P<0.0 \mathrm{I}, \mathrm{n}=5)$. Groups I, II, III, and IV indicate Ti + S. aureus, $\mathrm{NT}+\mathrm{S}$. aureus, NT-G $+\mathrm{S}$. aureus, and $\mathrm{Ti}+\mathrm{PBS}$, respectively. Ti, titanium without modification.

Abbreviations: CT, computed tomography; d, day; NT, nanotubes; NT-G, gentamicin-loaded nanotubes; PBS, phosphate-buffered saline; S. aureus, Staphylococcus aureus; w, weeks.

in the live bacteria and biofilm formation among the four groups. Considerably less green fluorescence and more discontinuous, scattered red fluorescence were observed in group III. An extraordinarily dense green fluorescence, which indicated biofilm formation, was observed in groups I and II; this feature is evidence of surviving adhered bacteria and biofilm formation in these two groups, and it was observed that there was denser green fluorescence in group I than in group II.

The results of the sonication after the rods were rolled over TSA further confirmed the detection mentioned before. As demonstrated in Figure 3C, the cultures obtained from the rods of group III $\left(1.57 \times 10^{4} \pm 2.08 \times 10^{3} \mathrm{CFU} / \mathrm{implant}\right)$ showed the lowest bacterial burden when compared 
A

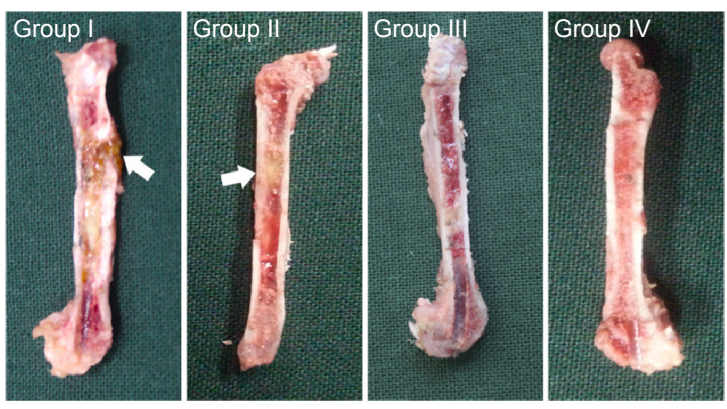

B

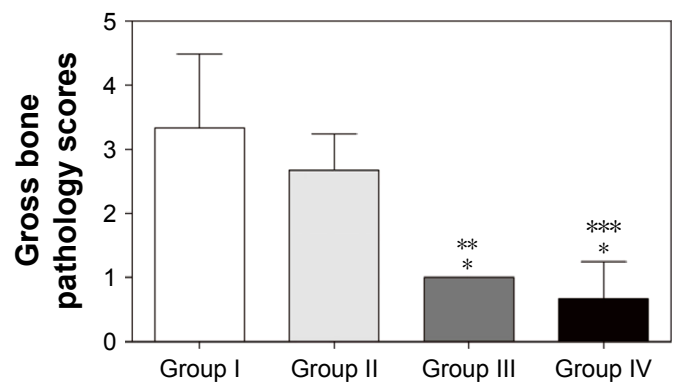

Figure 2 Gross appearance and scores of the left femur longitudinal sections at the time of sacrifice.

Notes: (A) Gross appearance. The white arrows mark intramedullary pus formation. (B) Gross bone pathology scores evaluation. The mean gross bone pathological scores of groups I through IV were 3.33 \pm I.16, 2.67 $\pm 0.58,1.00 \pm 0.00$, and $0.67 \pm 0.58$, respectively. *Denotes a significant difference compared with group I $(P<0.0$ I, $n=3)$. **Denotes a significant difference compared with group II $(P<0.05$, $\mathrm{n}=3)$. ****Denotes a significant difference compared with group $\|(P<0.0 I, n=3)$. Groups I, II, III, and IV indicate Ti + S. aureus, NT + S. aureus, NT-G + S. aureus, and $\mathrm{Ti}+\mathrm{PBS}$, respectively. $\mathrm{Ti}$, titanium without modification.

Abbreviations: NT, nanotubes; NT-G, gentamicin-loaded nanotubes; PBS, phosphate-buffered saline; S. aureus, Staphylococcus aureus.

with groups I $\left(6.70 \times 10^{5} \pm 7.55 \times 10^{4} \mathrm{CFU} /\right.$ implant $)$ and II $\left(4.60 \times 10^{5} \pm 6.24 \times 10^{4} \mathrm{CFU} /\right.$ implant $)(P<0.01)$. Group II also exhibited significantly lower CFU/implant compared with group I $(P<0.05)$. Meanwhile, the bacterial burden per gram of femur also demonstrated the following trend: group I, group II, and group III (Figure 3D); the lowest bacterial burden was observed in group III $\left(1.08 \times 10^{4} \pm 1.59 \times 10^{3} \mathrm{CFU} / \mathrm{g}\right)$ when compared with groups I $\left(5.08 \times 10^{5} \pm 8.91 \times 10^{4} \mathrm{CFU} / \mathrm{g}\right)$ and II $\left(3.44 \times 10^{5} \pm 6.56 \times 10^{4} \mathrm{CFU} / \mathrm{g}\right)(P<0.01)$. Group II also exhibited significantly lower $\mathrm{CFU} /$ femur than did group I $(P<0.05)$. Considering the bacterial log-reduction on the implants with respect to the Ti group, the bacteria found in the NT and NT-G groups were reduced by $0.16-\log$ and $1.63-$ $\log$, while on the femurs (per gram), the bacteria found in the NT and NT-G groups were reduced by $0.17-\log$ and 1.67-log, respectively. The SEM observations of the explanted implants further supported these results. As shown in Figure 4, dense biofilm formations were found on the implant surfaces in both groups I and II, but especially in group I. However, a few scattered bacteria exhibiting damaged morphologies were observed in group III. It is noteworthy that the nanotube coating on the titanium rods in groups II and III remained undamaged after the implantation, which verified the stability and feasibility of the nanotube structure.

\section{Histological evaluation}

The longitudinal and transverse decalcified sections from the different groups are demonstrated in Figures 5 and 6A, respectively. The morphological change on the left femur was assessed by hematoxylin and eosin and Masson's trichrome staining, while the bacterial residue was confirmed by Giemsa staining. In groups I and II, there was obvious destruction of cortical bone, which was accompanied by intracortical abscesses and inflammatory cell infiltration, medullary sequestrum formation and fibrosis, and many bacteria observed in the intramedullary cavities as shown by the Giemsa staining. However, relatively slight inflammatory cell infiltration and no evident bone destruction were demonstrated in group III. Meanwhile, there were Giemsa-stained colonized bacteria observed in bone tissues, but the bacteria numbers appeared to be dramatically reduced compared with those of groups I and II, which suggested that systemic antibiotic administration may have been necessary to eradicate the bacteria. In addition, no detectable signs of femur infection or bacteria were observed in group IV.

In addition, as described in Figure 6B, there was obvious bone destruction in groups I and II. Meanwhile, the implants in groups III and IV were in direct contact with the surrounding bone and elicited no obvious signs of cortical bone destruction or intramedullary inflammation at the boneimplant interface.

\section{Discussion}

The traditional remedy of an infected implant is a prolonged systemic antibiotic administration after device removal. ${ }^{10}$ The local use of antibiotic-loaded nanotubes has been widely reported to prevent this intractable clinical condition experimentally. ${ }^{15,17}$ Meanwhile, nanostructured surface topographies have been explored as effective approaches for enhancing desirable osteogenesis. ${ }^{40,41}$ As reported in our previous research, gentamicin-loaded nanotubes with diameters of $80 \mathrm{~nm}$ exhibited predictable drug release kinetics and significantly improved antimicrobial activity. ${ }^{16}$ In this study, we further investigated the effectiveness of gentamicin-loaded nanotubes on titanium surfaces to prevent implant-associated infections in a rat model.

The most common microorganisms correlated with implant-associated infection are S. aureus and S. epidermidis, which can adhere to implant surfaces and form biofims. ${ }^{15,42}$ 

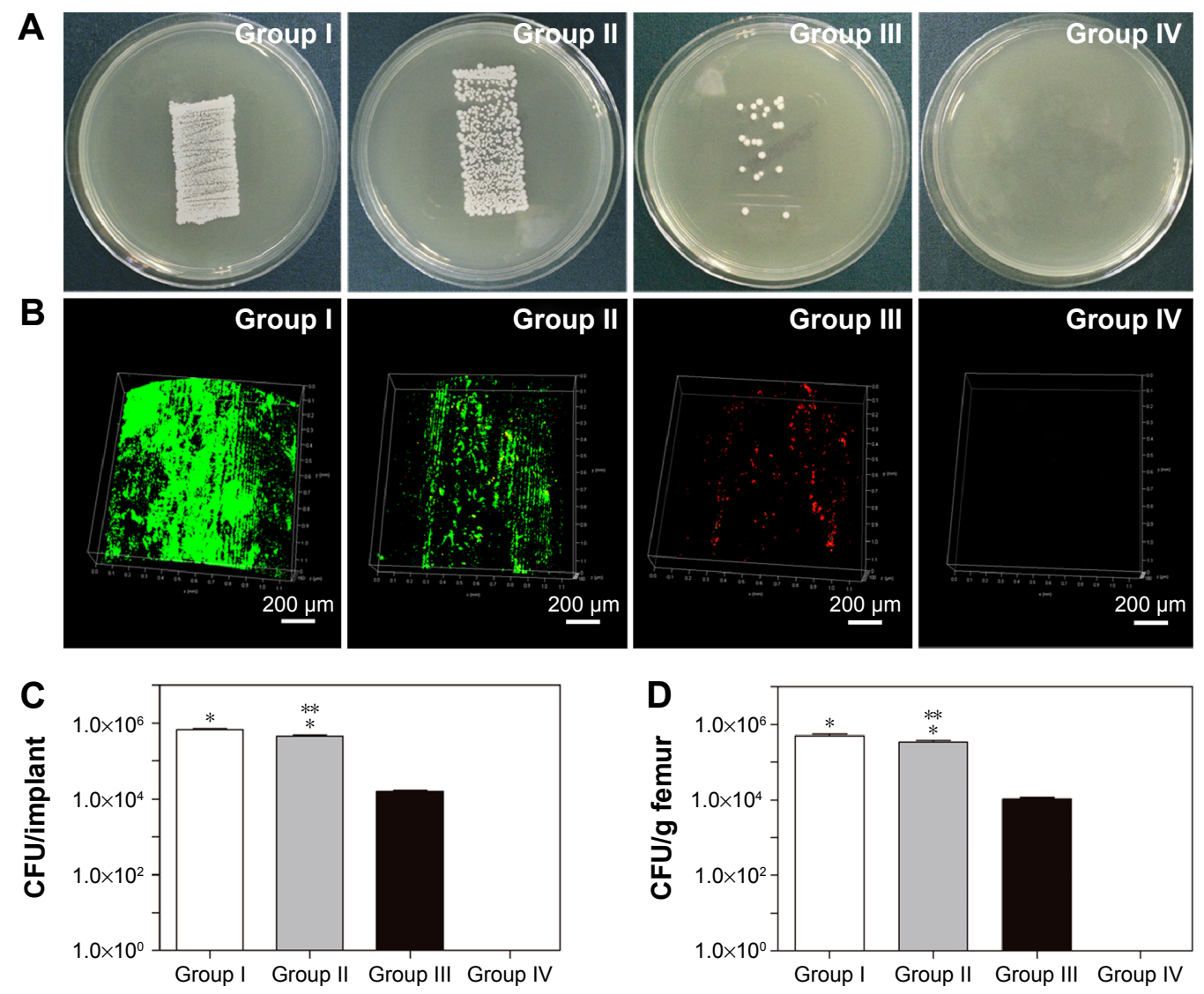

Figure 3 Microbiological evaluation of the implants and bones.

Notes: (A) Rollover cultures obtained from the explanted rods. (B) Confocal laser scanning microscopy observations of the explanted rods. The live bacteria were stained with SYTO 9 and fluorescence green, and the dead bacteria were stained with propidium iodide and fluorescence red. The magnification is $\times 100$. (C) Number of detached adhered bacteria and amount of biofilm after the rods were rolled over TSA. (D) Quantity of CFU/g of pulverized femur. *Denotes a significant difference compared with groups III and IV $(P<0.0$ I, $n=3)$. **Denotes a significant difference compared with group I $(P<0.05, n=3)$. Groups I, II, III, and IV indicate Ti + S. aureus, NT + S. aureus, NT-G $+\mathrm{S}$. aureus, and $\mathrm{Ti}+\mathrm{PBS}$, respectively. $\mathrm{Ti}$, titanium without modification.

Abbreviations: NT, nanotubes; NT-G, gentamicin-loaded nanotubes; PBS, phosphate-buffered saline; S. aureus, Staphylococcus aureus; TSA, tryptone soy agar; CFU, colonyforming unit.

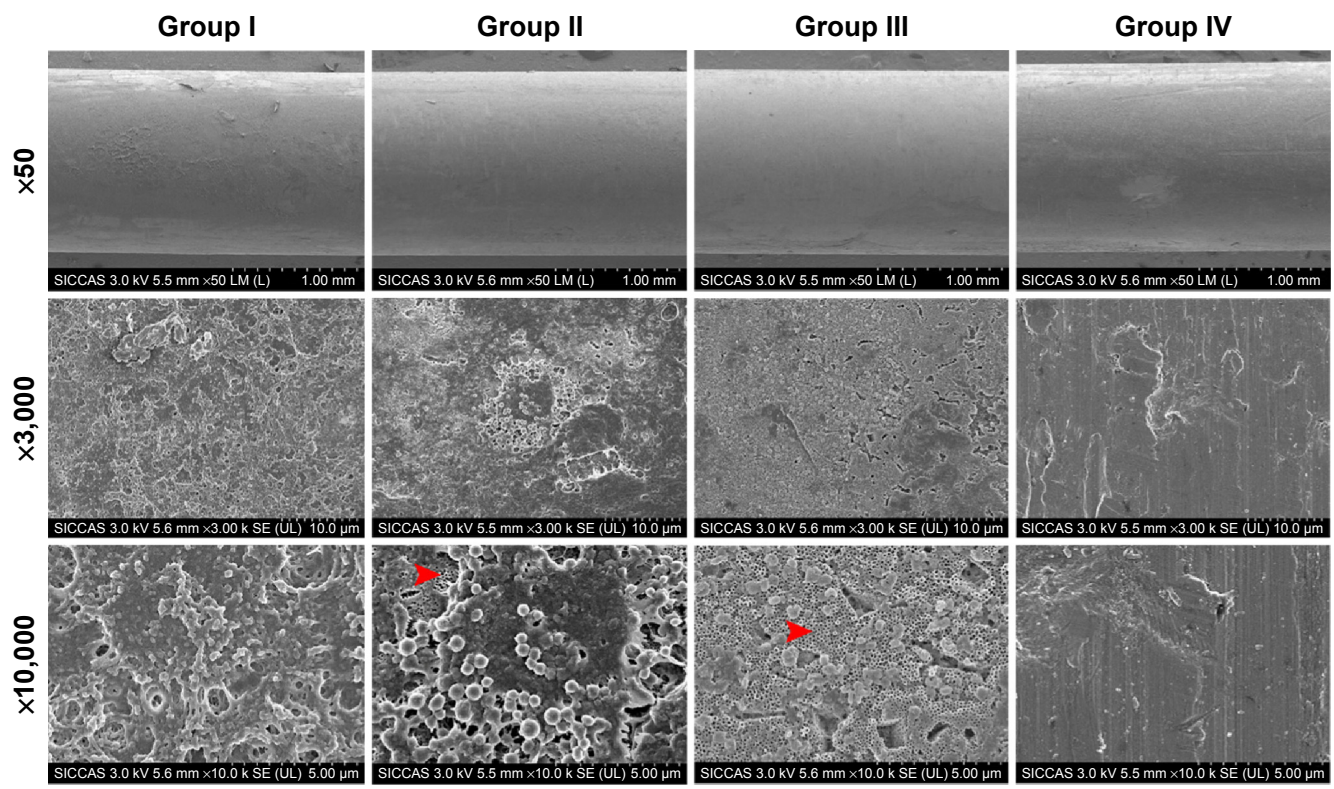

Figure 4 Scanning electron microscopy (SEM) observations of the explanted implants.

Notes: The red arrowheads indicate the intact nanotubular structure on the titanium rods. The magnifications are $\times 50, \times 3,000$, and $\times 10,000$, and the scale bars are $1.0 \mathrm{~mm}, 10.0 \mu \mathrm{m}$, and $5.0 \mu \mathrm{m}$, respectively. Groups I, II, III, and IV indicate Ti + S. aureus, NT + S. aureus, NT-G + S. aureus, and Ti + PBS, respectively. Ti, titanium without modification. Abbreviations: NT, nanotubes; NT-G, gentamicin-loaded nanotubes; PBS, phosphate-buffered saline; S. aureus, Staphylococcus aureus. 


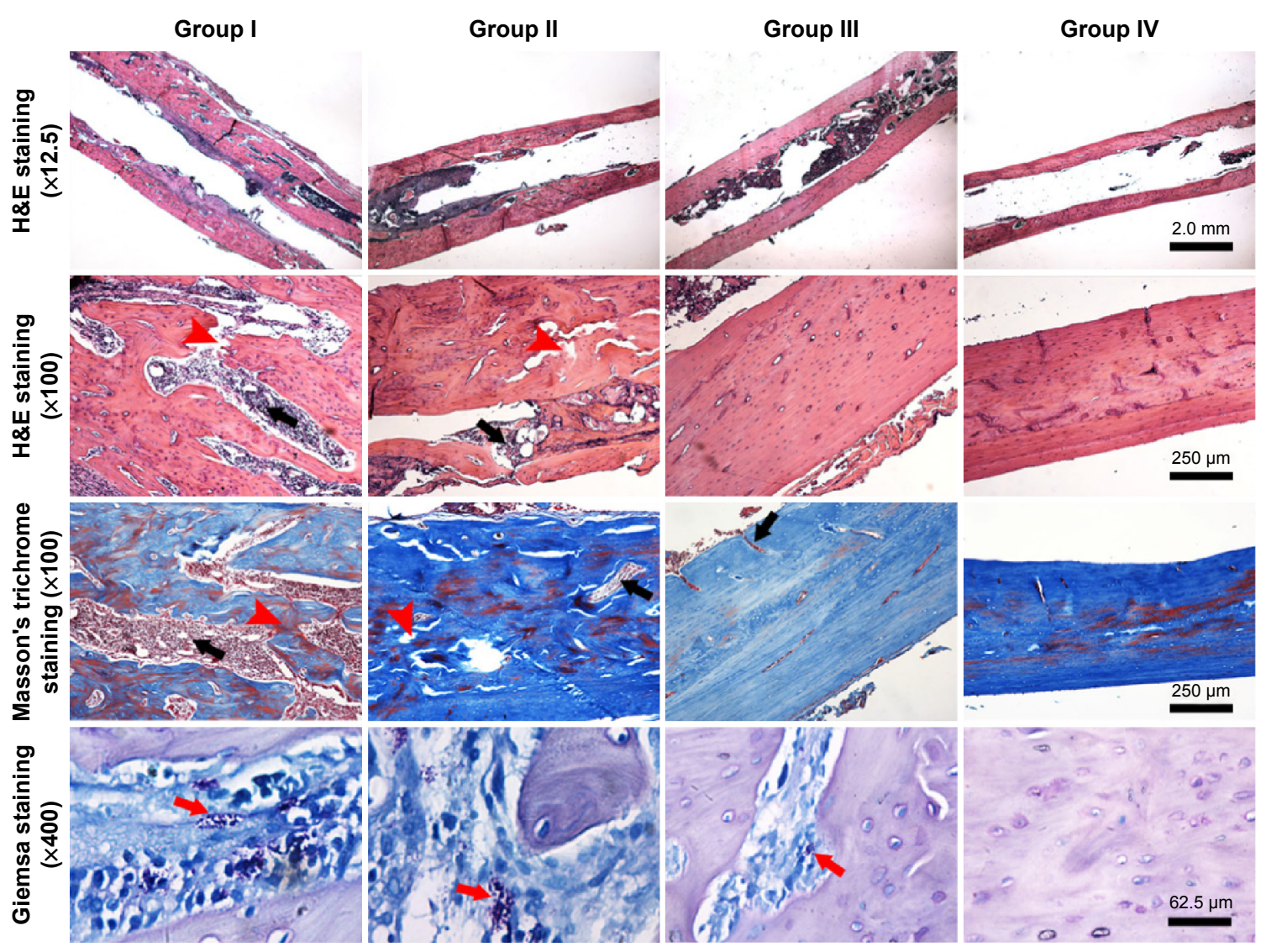

Figure 5 Representative histological images of longitudinal sections from middle femur with H\&E staining, Masson's trichrome staining, and Giemsa staining at 4 weeks after implantation.

Notes: The black arrows indicate intracortical abscesses or inflammatory cells; the red arrowheads indicate bone cortex destruction; and the red arrows indicate bacteria. Groups I, II, III, and IV indicate Ti + S. aureus, NT + S. aureus, NT-G + S. aureus, and Ti + PBS, respectively. Ti, titanium without modification.

Abbreviations: H\&E, hematoxylin and eosin; NT, nanotubes; NT-G, gentamicin-loaded nanotubes; PBS, phosphate-buffered saline; S. aureus, Staphylococcus aureus.

In this present in vivo study, a bacterial suspension of S. aureus (ATCC 25923) was injected into the left distal femoral medullary cavity of each rat prior to the implantation of a titanium implant. The validity of this bone infection model was confirmed by the X-ray images at 3 weeks and 6 weeks postsurgery, which were consistent with previous research. ${ }^{36,39}$ Subsequently, physical examination, radiographic evaluation, gross bone pathology, microbiological evaluation, and histological assessment were used for the comparison of efficacy between the NT-G, Ti, and NT groups in the treatment of intramedullary implant-related infections. Our in vivo observations demonstrated that, in all animals, body weights increased gradually and temperatures did not fluctuate; these observations indicated that the infections were localized around the femurs. Meanwhile, the X-ray and micro-CT images obtained from the NT-G group did not exhibit obvious signs of articular surface destruction, osteolysis, or periosteal reaction. Moreover, significantly lower radiographic and gross bone scores were also observed in the NT-G group when compared with those of the Ti and NT groups. Combined with the microbiological and histological results, these results show the bacterial inhibition potential of NT-G coatings in the development of implant-associated infections.

Various in vitro studies have demonstrated that $80 \mathrm{~nm}$ diameter titanium nanotubes exhibit good antibacterial activity; ${ }^{16,26,27}$ however, our in vivo observations revealed that the NT coating alone displayed limited antibacterial capability over the course of 6 weeks postsurgery. In the NT group, obvious osteolysis and slight periosteal reactions were observed in the X-ray images after 3 weeks postsurgery, with increases in articular surface damage, osteosclerosis, and deformities at 6 weeks, as analyzed by micro-CT. Meanwhile, microbiological evaluation showed bacterial loads in the following trend: Ti $>$ NT $>$ NT-G. Although the antibacterial activity of NT was significantly better than that of $\mathrm{Ti}$, it was still insufficient to prevent the development of bone infection in vivo when compared with NT-G. Once adhered bacteria are not killed effectively, a biofilm will irreversibly evolve, ${ }^{43}$ and the biofilm bacteria will be 1,000 times stronger than the planktonic bacteria because of the physical protective effects of the biofilm matrix. ${ }^{44}$ Therefore, a relatively promising approach is to 


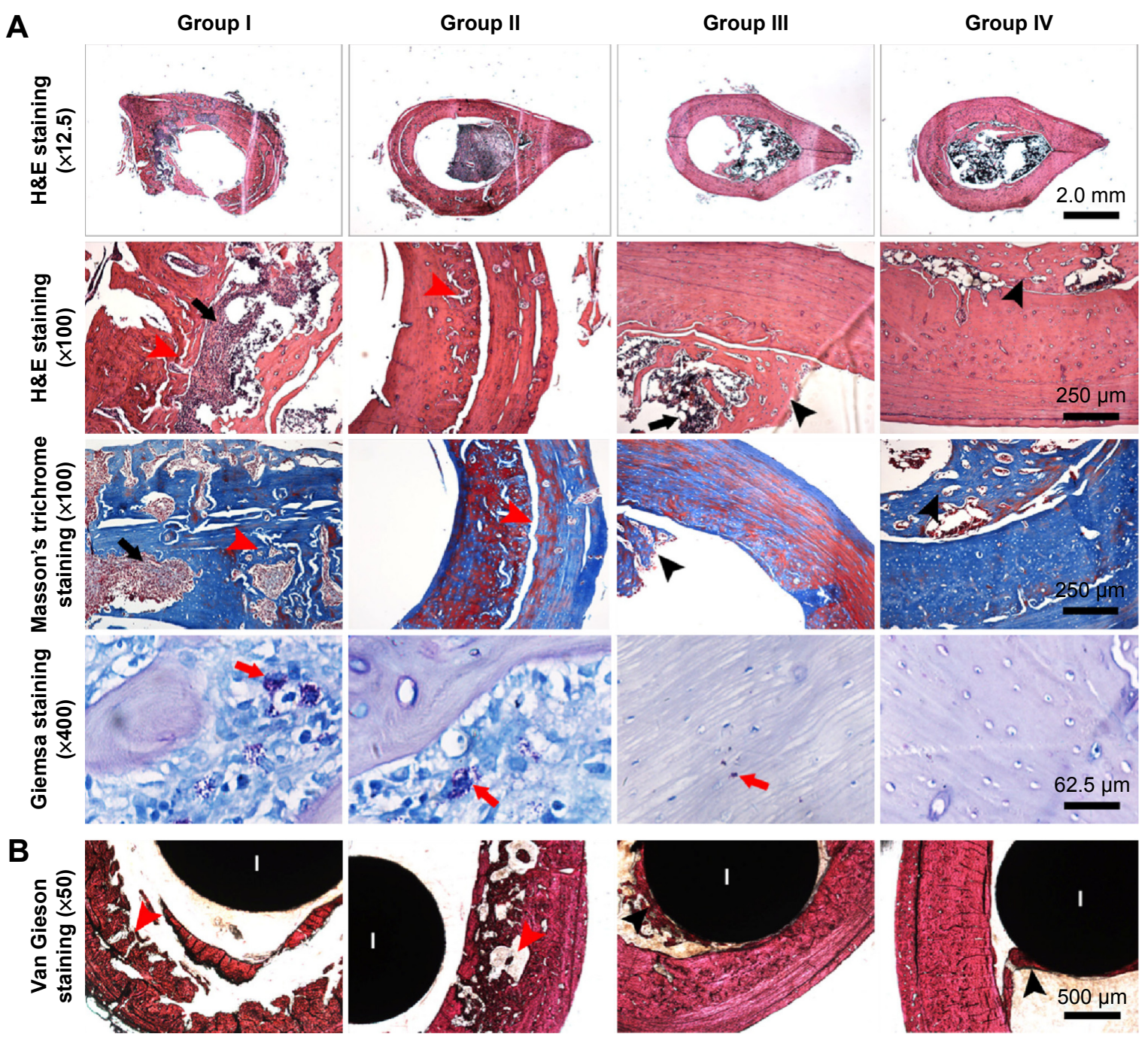

Figure 6 Representative histological images of transverse sections from the middle femur at 4 weeks after implantation.

Notes: (A) Decalcified sections without implants with H\&E staining, Masson's trichrome staining, and Giemsa staining. (B) Van Gieson-stained undecalcified sections with implants. The black arrows indicate intracortical abscesses or inflammatory cells; the red arrowheads indicate bone cortex destruction; the red arrows indicate bacteria; and the black arrowheads indicate new bone formation around the implants. Groups I, II, III, and IV indicate Ti + S. aureus, NT + S. aureus, NT-G + S. aureus, and Ti + PBS, respectively. Ti, titanium without modification.

Abbreviations: H\&E, hematoxylin and eosin; I, implant; NT, nanotubes; NT-G, gentamicin-loaded nanotubes; PBS, phosphate-buffered saline; S. aureus, Staphylococcus aureus.

prevent bacterial adhesion at the time of implantation prior to biofilm formation; the prognosis of implant-associated infections will then depend on biofilm formation inhibition rather than elimination in animal models. ${ }^{27}$

As demonstrated in the SEM observation of explanted rods, the nanotubular structure on the titanium rods in groups II and III remained undamaged after the implantation, which indicated the in vivo stability of the nanotubes, and therefore provides reliable evidence for the viability of the drug-loaded nanotubes implant in orthopedic surgeries. The microbiological evaluation revealed that the cultures obtained from the implants and femurs in the NT-G group had the lowest bacterial burden when compared with those of the $\mathrm{Ti}$ and NT groups. Therefore, additional treatment with systemic antibiotic administration may be necessary to completely eradicate the bacteria. However, residual bacteria detected in group III may also be attributed to the development of antimicrobial resistance. According to our previous in vitro study, the total amounts of gentamicin released from nanotubes with a diameter of $80 \mathrm{~nm}$ over 57 hours was $91.45 \mu \mathrm{g},{ }^{16}$ which was above the minimum inhibitory concentrations of the tested strain $(1 \mu \mathrm{g} / \mathrm{mL})$. Furthermore, as the drug-loading release capacity can be flexibly tuned by monitoring nanotube structure parameters such as the diameter and length at various anodization conditions and by implant size, ${ }^{9}$ the fabrication of antibiotic-loaded nanotubes with various different structure parameters may be a valuable approach to achieve optimal effectiveness of such an in vivo anti-infection model. 


\section{Conclusion}

In general, this effective in vivo study showed that the NT-G group exhibited significant bacterial inhibition when compared with the Ti and NT groups in this $S$. aureus infection rat model. The NT coatings also resulted in alleviated bacterial burdens and therefore demonstrated the feasibility of the clinical application of this antibiotic-loaded titanium nanotube-based implant for combating orthopedic implantassociated infection.

\section{Acknowledgments}

This research was financially supported by the National Natural Science Foundation of China (Nos 31271015, 81501856), the Shanghai Science and Technology Development Fund (13JC1403900, 13DZ2294000), and the Medical Engineering Collaborative Project of Shanghai Jiao Tong University (YG2014ZD01).

\section{Disclosure}

The authors report no conflicts of interest in this work.

\section{References}

1. Darouiche RO. Treatment of infections associated with surgical implants. N Engl J Med. 2004;350(14):1422-1429.

2. Trebse R, Pisot $\mathrm{V}$, Trampuz $\mathrm{A}$. Treatment of infected retained implants. J Bone Joint Surg Br. 2005;87(2):249-256.

3. Lee KJ, Goodman SB. Identification of periprosthetic joint infection after total hip arthroplasty. J Orthop Translat. 2015;3(1):21-25.

4. Long M, Rack HJ. Titanium alloys in total joint replacement - a materials science perspective. Biomaterials. 1998;19(18):1621-1639.

5. Ferrigno N, Laureti M, Fanali S, Grippaudo G. A long-term follow-up study of non-submerged ITI implants in the treatment of totally edentulous jaws. Part I: ten-year life table analysis of a prospective multicenter study with 1286 implants. Clin Oral Implants Res. 2002;13(3): 260-273.

6. Zhao L, Chu PK, Zhang Y, Wu Z. Antibacterial coatings on titanium implants. J Biomed Mater Res B Appl Biomater. 2009;91(1): 470-480.

7. Jin $\mathrm{G}$, Qin $\mathrm{H}$, Cao $\mathrm{H}$, et al. Synergistic effects of dual $\mathrm{Zn} / \mathrm{Ag}$ ion implantation in osteogenic activity and antibacterial ability of titanium. Biomaterials. 2014;35(27):7699-7713.

8. Young S, Lie SA, Hallan G, Zirkle LG, Enqesæter LB, Havelin LI. Risk factors for infection after 46,113 intramedullary nail operations in low and middle-income countries. World J Surg. 2013;37(2):349-355.

9. Gulati K, AW MS, Losic D. Drug-eluting Ti wires with titania nanotube arrays for bone fixation and reduced bone infection. Nanoscale Res Lett. 2011;6:571.

10. Birdsall PD, Milne DD. Toxic shock syndrome due to percutaneous Kirschner wires. Injury. 1999;30(7):509-510.

11. Hargreaves DG, Drew SJ, Eckersley R. Kirschner wire pin tract infection rates: a randomized controlled trial between percutaneous and buried wires. J Hand Surg Br. 2004;29(4):374-376.

12. Zwilling V, Darque-Ceretti E, Boutry-Forveille A. Structure and physicochemistry of anodic oxide films on titanium and TA6V alloy. Surf Interface Anal. 1999;27:629-637.

13. Popat KC, Eltgroth M, LaTempa TJ, Grimes CA, Desai TA. Decreased Staphylococcus epidermis adhesion and increased osteoblast functionality on antibiotic-loaded titania nanotubes. Biomaterials. 2007; 28(32):4880-4888.
14. Eaninwene G 2nd, Yao C, Webster TJ. Enhanced osteoblast adhesion to drug-coated anodized nanotubular titanium surfaces. Int J Nanomedicine. 2008;3(2):257-264.

15. Losic D, Aw MS, Santos A, Gulati K, Bariana M. Titania nanotube arrays for local drug delivery: recent advances and perspectives. Expert Opin Drug Deliv. 2015;12(1):103-127.

16. Lin WT, Tan HL, Duan ZL, et al. Inhibited bacterial biofilm formation and improved osteogenic activity on gentamicin-loaded titania nanotubes with various diameters. Int J Nanomedicine. 2014;9:1215-1230.

17. Zhang H, Sun Y, Tian A, et al. Improved antibacterial activity and biocompatibility on vancomycin-loaded $\mathrm{TiO} 2$ nanotubes: in vivo and in vitro studies. Int J Nanomedicine. 2013;8:4379-4389.

18. Ma M, Kazemzadeh-Narbat M, Hui Y, et al. Local delivery of antimicrobial peptides using self-organized $\mathrm{TiO} 2$ nanotube arrays for peri-implant infections. J Biomed Mater Res A. 2012;100(2): $278-285$.

19. Chennell P, Feschet-Chassot E, Devers T, Awitor KO, Descamps S, Sautou V. In vitro evaluation of $\mathrm{TiO} 2$ nanotubes as cefuroxime carriers on orthopaedic implants for the prevention of periprosthetic joint infections. Int J Pharm. 2013;455(1-2):298-305.

20. Zhao L, Wang H, Huo K, et al. Antibacterial nano-structured titania coating incorporated with silver nanoparticles. Biomaterials. 2011; 32(24):5706-5716.

21. Gomathi Devi L, Naqaraj B. Disinfection of Escherichia coli gram negative bacteria using surface modified $\mathrm{TiO}_{2}$ : optimization of $\mathrm{Ag}$ metallization and depiction of charge transfer mechanism. Photochem Photobiol. 2014;90(5):1089-1098.

22. Li Y, Xiong W, Zhang C, et al. Enhanced osseointegration and antibacterial action of zinc-loaded titania-nanotube-coated titanium substrates: in vitro and in vivo studies. $J$ Biomed Mater Res A. 2014;102(11): 3939-3950.

23. Hang R, Gao A, Huang X, et al. Antibacterial activity and cytocompatibility of Cu-Ti-O nanotubes. J Biomed Mater Res A. 2014;102(6): $1850-1858$.

24. Gao A, Hang R, Huang X, et al. The effects of titania nanotubes with embedded silver oxide nanoparticles on bacteria and osteoblasts. Biomaterials. 2014;35(13):4223-4235.

25. Neoh KG, Hu X, Zheng D, Kang ET. Balancing osteoblast functions and bacterial adhesion on functionalized titanium surfaces. Biomaterials. 2012;33(10):2813-2822.

26. Peng Z, Ni J, Zheng K, et al. Dual effects and mechanism of $\mathrm{TiO}_{2}$ nanotube arrays in reducing bacterial colonization and enhancing C3H10T1/2 cell adhesion. Int J Nanomedicine. 2013;8:3903-3105.

27. Ercan B, Taylor E, Alpaslan E, Webster TJ. Diameter of titanium nanotubes influences anti-bacterial efficacy. Nanotechnology. 2011;22(29): 295102.

28. Wang $\mathrm{N}, \mathrm{Li} \mathrm{H}, \mathrm{Lu} \mathrm{W}$, et al. Effects of $\mathrm{TiO}_{2}$ nanotubes with different diameters on gene expression and osseointegration of implants in minipigs. Biomaterials. 2011;32(29):6900-6911.

29. Lucke M, Schmidmaier G, Sadoni S, et al. Gentamicin coating of metallic implants reduces implant-related osteomyelitis in rats. Bone. 2003;32(5):521-531.

30. Puckett SD, Taylor R, Raimondo T, Webster TJ. The relationship between the nanostructure of titanium surfaces and bacterial attachment. Biomaterials. 2010;31(4):706-713.

31. Correa CB, Pires JR, Fernandes-Filho RB, Sartori R, Vaz LG. Fatigue and fluoride corrosion on Streptococcus mutans adherence to titanium-based implant/component surfaces. J Prosthodont. 2009;18(5):382-387.

32. Qin H, Zhao YC, An ZQ, et al. Enhanced antibacterial properties, biocompatibility, and corrosion resistance of degradable $\mathrm{Mg}-\mathrm{Nd}-\mathrm{Zn}-\mathrm{Zr}$ alloy. Biomaterials. 2015;53:211-220.

33. Ni JH, Frandsen CJ, Chen LH, et al. Fabrication of gradient TiO2 nanotubes on Ti foil by anodization. Adv Eng Mater. 2013;15(6): 464-468.

34. Tan X, Qin N, Wu C, et al. Transcriptome analysis of the biofilm formed by methicillin susceptible Staphylococcus aureus. Sci Rep. 2015; 5:11997. 
35. Cole AM, Weis P, Diamond G. Isolation and characterization of pleurocidin, an antimicrobial peptide in the skin secretions of winter flounder. J Biol Chem. 1997;272(18):12008-12013.

36. Tan HL, Ao HY, Ma R, Lin WT, Tang TT. In vivo effect of quaternized chitosan-loaded polymethylmethacrylate bone cement on methicillinresistant Staphylococcus epidermidis infection of the tibial metaphysis in a rabbit model. Antimicrob Agents Chemother. 2014;58(10): 6016-6023.

37. Rissing JP, Buxton TB, Weinstein RS, Shockley RK. Model of experimental chronic osteomyelitis in rats. Infect Immun. 1985;47(3): 581-586.

38. Petty W, Spanier S, Shuster JJ, Sliverthorne C. The influence of skeletal implants on incidence of infection: experiments in a canine model. J Bone Joint Surg Am. 1985;67(8):1236-1244.

39. Alt V, Bitschnau A, Osterling J, et al. The effects of combined gentamicin-hydroxyapatite coating for cementless joint prostheses on the reduction of infection rates in a rabbit infection prophylaxis model. Biomaterials. 2006;27(26):4627-4634.
40. Lin LW, Wang H, Ni M, et al. Enhanced osteointegration of medical titanium implant with surface modifications in micro/nanoscale structures. J Orthop Translat. 2014;2(1):35-42.

41. Gong T, Xie J, Liao JF, Zhang T, Lin SY, Lin TF. Nanomaterials and bone regeneration. Bone Res. 2015;3:15029.

42. Barth E, Myrvik QM, Waqner W, Gristina AG. In vitro and in vivo comparative colonization of Staphylococcus aureus and Staphylococcus epidermidis on orthopaedic implant materials. Biomaterials. 1989; 10(5):325-328.

43. Ariza J, Euba G, Murillo O. Infecciones relacionadas con las prótesis articulares. [Orthopedic device-related infections]. Enferm Infecc Microbiol Clin. 2008;26(6):380-390. Spanish.

44. Smith AW. Biofilms and antibiotic therapy: is there a role for combating bacterial resistance by the use of novel drug delivery systems? $A d v$ Drug Deliv Rev. 2005;57(10):1539-1550.
International Journal of Nanomedicine

\section{Publish your work in this journal}

The International Journal of Nanomedicine is an international, peerreviewed journal focusing on the application of nanotechnology in diagnostics, therapeutics, and drug delivery systems throughout the biomedical field. This journal is indexed on PubMed Central, MedLine, CAS, SciSearch ${ }^{\circledR}$, Current Contents ${ }^{\circledR} /$ Clinical Medicine,

\section{Dovepress}

Journal Citation Reports/Science Edition, EMBase, Scopus and the Elsevier Bibliographic databases. The manuscript management system is completely online and includes a very quick and fair peer-review system, which is all easy to use. Visit http://www.dovepress.com/ testimonials.php to read real quotes from published authors. 